\title{
Predictors of pacing-dependency in patients with cardiovascular implantable electronic devices
}

\author{
Wolfram Grimm ${ }^{1}$, Kathrin Grimm ${ }^{1}$, Brandon Greene ${ }^{2}$, Mariana Parahuleva ${ }^{1}$ \\ ${ }^{1}$ Department of Cardiology, University Hospital of Marburg and Gießen, \\ Philipps-University Marburg, Marburg, Germany \\ ${ }^{2}$ Institute for Medical Bioinformatics and Biostatistics, \\ Philipps-University Marburg, Marburg, Germany
}

\begin{abstract}
Background: Data on the prevalence and predictors for the development of pacing-dependency in patients with cardiovascular implantable electronic devices (CIEDs) are sparse.

Methods: Pacing-dependency defined as an absence of intrinsic rhythm of $\geq 30 \mathrm{bpm}$ was determined in 802 consecutive patients with CIEDs who visited the documented pacemaker or implantable cardioverter-defibrillator outpatient clinic for routine follow-up.

Results: A total of 131 (16\%) patients were found to be pacing-dependent $67 \pm 70$ months after CIED implant. Multivariate analysis revealed a significant association between pacing-dependency and the following clinical variables: second or third-degree atrioventricular $(A V)$ block at implant (OR $=19.9 ; 95 \%$ CI: $10.9-38.5, p<0.01)$, atrial fibrillation at implant $(O R=2.15 ; 95 \%$ CI: $1.16-4.05, p=0.02)$, left ventricular ejection fraction $(L V E F) \leq 30 \%(O R=2.06 ; 95 \% C I: 1.03-4.15, p=0.04), B$-type natriuretic peptide $(B N P)>150 \mathrm{pg} / \mathrm{mL}(\mathrm{OR}=2.12 ; 95 \%$ CI: 1.16-3.97, $p=0.02)$, chronic kidney disease (OR $=1.86 ; 95 \%$ CI: $1.08-3.26, p=0.03)$, and follow-up duration after implantation $>5$ years $(O R=3.29$; 95\% CI: 1.96-5.64, $p<0.01$ ). None of the remaining clinical variables including age, gender, diabetes mellitus, underlying heart disease, prior cardiac surgery or medication during follow-up including betablockers and amiodarone predicted pacing-dependency.

Conclusions: Pacing-dependency is associated with second or third-degree AV-block at implant, atrial fibrillation before implant, low LVEF, elevated BNP, chronic kidney disease and follow-up duration after implant. (Cardiol J 2021; 28, 3: 423-430)
\end{abstract}

Key words: pacing-dependency, permanent pacemaker, implantable cardioverter-defibrillator

\section{Introduction}

Knowledge of pacing-dependency following implantation of cardiovascular implantable electronic devices (CIEDs) is very important in various clinical settings including elective generator change, potential electromagnetic interference and management of suspected lead or generator malfunction $[1,2]$. Although several million permanent pacemakers and implantable cardioverter-defibrillators (ICDs) with bradycardia pacing capability have been implanted 60 years after the first pacemaker implantation in 1958, few studies have investigated the prevalence of pacing dependency and clinical predictors for the development of pacing dependency [3-17]. Thus, the aim herein was to determine prevalence and predictors of pacing dependency in a well-defined cohort of 802 patients with pacemakers or ICDs at the documented clinic.

Address for correspondence: Wolfram Grimm, MD, Department of Cardiology, Philipps-University Marburg,

Baldingerstraße, 35033 Marburg, Germany, tel: +49-6421-586-9748, fax: +49-6421-586-8954,

e-mail: grimmwk.rauschenberg@t-online.de

Received: 1.04.2019 Accepted: 26.08.2019

This article is available in open access under Creative Common Attribution-Non-Commercial-No Derivatives 4.0 International (CC BY-NC-ND 4.0) license, allowing to download articles and share them with others as long as they credit the authors and the publisher, but without permission to change them in any way or use them commercially. 


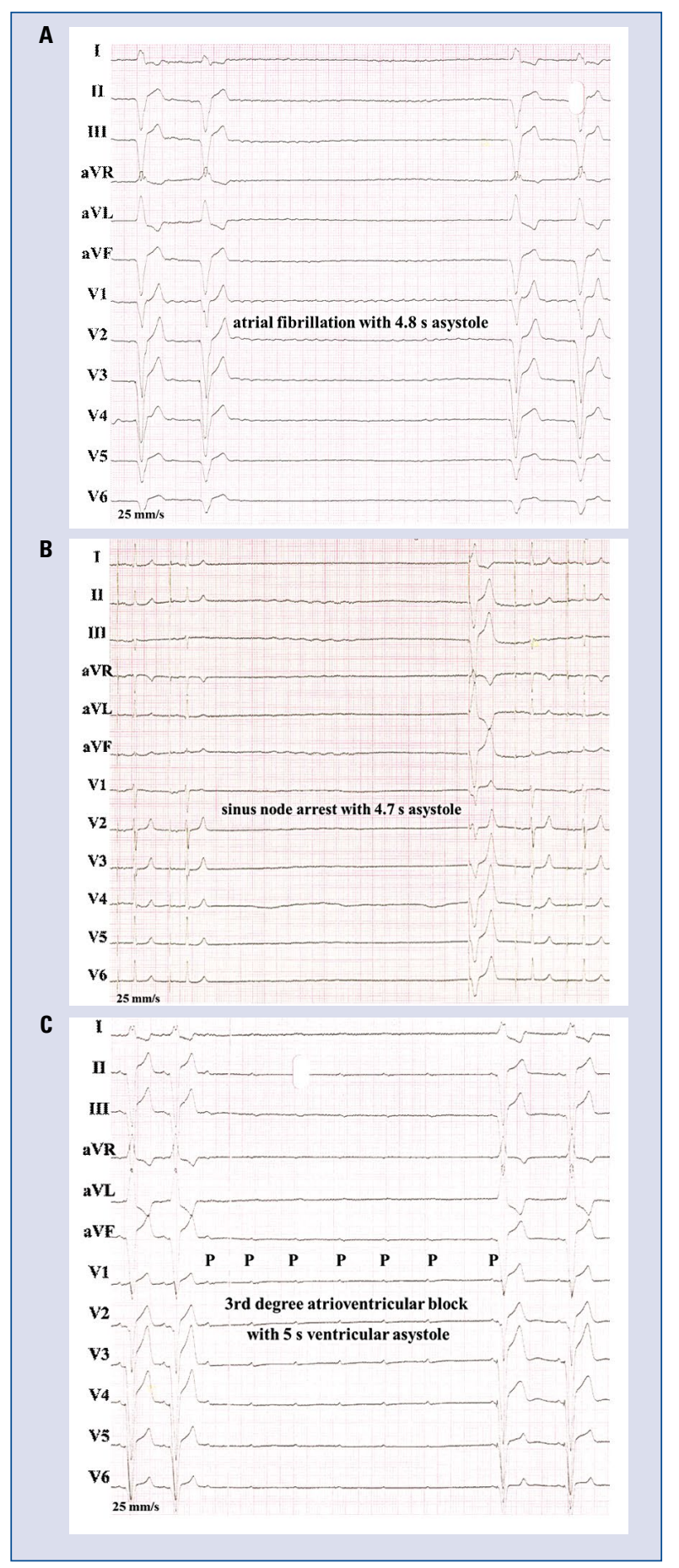

Figure 1. Electrocardiogram recordings with a paper speed of $25 \mathrm{~mm} / \mathrm{s}$ showing typical examples of ventricular asystole following transient inhibition of pacing; A. Ventricular asystole due to third degree atrioventricular block (AVB) without ventricular escape rhythm in a patient with permanent atrial fibrillation; B. Ventricular asystole due to sinus arrest without escape rhythm in a patient with sinus node disease; C. Ventricular asystole due to third degree AVB without ventricular escape rhythm in a patient with second degree AVB type Mobitz at the time of pacemaker implant.

\section{Methods}

\section{Study population}

After written informed consent had been obtained, pacing dependency was determined prospectively in 802 consecutive patients who came to the pacemaker and ICD outpatient clinic for routine follow-up between January 2018 and December 2018 and in whom a permanent pacemaker or ICD had been implanted for at least 6 months by this institution. Pacing dependency was defined as an absence of intrinsic rhythm $\geq 30 \mathrm{bpm}$ after lowering the pacing rate to $30 \mathrm{bpm}$ for at least $10 \mathrm{~s}$ or after transient inhibition of pacing therapy (Fig. 1). A high-degree atrioventricular (AV) block at implant was defined as second degree AV block type Mobitz or third-degree AV block. Chronic kidney disease (CKD) of at least stage 3 was diagnosed in the presence of at least two estimated glomerular filtration rates (eGFR) using the Modification of Diet in Renal Disease formula below $60 \mathrm{~mL} / \mathrm{min}$ per $1.73 \mathrm{~m}^{2}$ with an interval of at least 3 months. The study protocol was reviewed and approved by the ethics committee of the Philipps-University of Marburg.

\section{Statistical analysis}

Results are expressed as mean \pm standard deviation (SD) for continuous variables with normal distribution and median values with interquartile range (IQR) for continuous variables without normal distribution. Univariate comparisons of clinical characteristics between patients with and without pacing dependency were performed using the Student t-test or the Mann-Whitney U test for continuous variables, categorical values were compared using $\chi^{2}$ and the Fisher exact tests, where appropriate. Logistic regression analysis was used to generate a multivariate model including all potential predictors of pacing dependency listed in Table 1 in order to investigate which factors showed independent effects on the risk of developing pacemaker dependency after adjustment for confounding by other factors including the presence or absence of ICD therapy as well as cardiac resynchronization therapy (CRT). All probability values reported are two-sided, and a probability value of $p<0.05$ was considered to indicate statistical significance. R-software version 3.5.0 (www.R-project.org) was used for statistical analyses.

\section{Results}

\section{Clinical characteristics}

The clinical characteristics of 802 study patients are summarized in Table 1 and included 563 
Table 1. Clinical characteristics of 802 patients with and without pacing dependency.

\begin{tabular}{|c|c|c|c|c|}
\hline \multirow[t]{2}{*}{ Clinical variable } & \multirow{2}{*}{$\begin{array}{c}\text { All patients } \\
N=802\end{array}$} & \multicolumn{2}{|c|}{ Pacing-dependency } & \multirow[t]{2}{*}{$\mathbf{P}$} \\
\hline & & Yes $(n=131)$ & No $(n=671)$ & \\
\hline Age [years] & $74 \pm 13$ & $75 \pm 12$ & $74 \pm 13$ & 0.23 \\
\hline Male gender & $521(65 \%)$ & $89(68 \%)$ & $432(64 \%)$ & 0.50 \\
\hline Body mass index $\left[\mathrm{kg} / \mathrm{m}^{2}\right]$ & $28 \pm 12$ & $28 \pm 5$ & $28 \pm 13$ & 0.86 \\
\hline Arterial hypertension & $622(78 \%)$ & $105(80 \%)$ & $517(77 \%)$ & 0.27 \\
\hline Diabetes mellitus & $143(18 \%)$ & $19(15 \%)$ & $124(18 \%)$ & 0.43 \\
\hline Atrial fibrillation before implant & $242(30 \%)$ & $38(29 \%)$ & $205(31 \%)$ & 0.78 \\
\hline Left bundle branch block at implant & $141(18 \%)$ & $25(19 \%)$ & $116(17 \%)$ & 0.62 \\
\hline Chronic kidney disease & $330(41 \%)$ & $70(53 \%)$ & $260(39 \%)$ & $<0.01$ \\
\hline \multicolumn{5}{|l|}{ Heart failure severity } \\
\hline Left ventricular ejection fraction $\leq 30 \%$ & $179(22 \%)$ & $41(31 \%)$ & $138(21 \%)$ & $<0.01$ \\
\hline NYHA functional class III or IV & $320(40 \%)$ & $74(56 \%)$ & $246(37 \%)$ & $<0.01$ \\
\hline B-type natriuretic peptide $>150 \mathrm{pg} / \mathrm{mL}^{\mathrm{a}}$ & $408(57 \%)$ & $77(71 \%)$ & $331(54 \%)$ & $<0.01$ \\
\hline Underlying cardiac disease & & & & 0.07 \\
\hline Coronary artery disease & $313(39 \%)$ & $45(34 \%)$ & $268(40 \%)$ & \\
\hline Nonischemic dilated cardiomyopathy & $119(15 \%)$ & $26(20 \%)$ & $93(14 \%)$ & \\
\hline Hypertensive heart disease & $180(22 \%)$ & $35(31 \%)$ & $145(22 \%)$ & \\
\hline Valvular heart disease & $101(13 \%)$ & $17(15 \%)$ & $84(13 \%)$ & \\
\hline Other cardiac diseases ${ }^{b}$ & $13(2 \%)$ & $4(4 \%)$ & $9(1 \%)$ & \\
\hline No structural heart disease & $76(9 \%)$ & $4(4 \%)$ & $72(11 \%)$ & \\
\hline \multicolumn{5}{|l|}{ Previous cardiac surgery } \\
\hline Aortocoronary bypass grafting & $95(12 \%)$ & $17(13 \%)$ & $78(12 \%)$ & 0.77 \\
\hline Surgical aortic valve replacement & $29(4 \%)$ & $4(3 \%)$ & $25(4 \%)$ & 0.90 \\
\hline Mitral valve reconstruction or replacement & $15(2 \%)$ & $1(1 \%)$ & $14(2 \%)$ & 0.50 \\
\hline Transcatheter aortic valve replacement & $43(5 \%)$ & $9(7 \%)$ & $34(5 \%)$ & 0.53 \\
\hline \multicolumn{5}{|l|}{ Cardiovascular implantable electronic device } \\
\hline Antibradycardia pacemaker & $563(70 \%)$ & $103(79 \%)$ & $460(69 \%)$ & 0.02 \\
\hline Implantable cardioverter-defibrillator & $239(30 \%)$ & $28(21 \%)$ & $211(31 \%)$ & 0.02 \\
\hline Cardiac resynchronization therapy device & $93(12 \%)$ & $20(15 \%)$ & $73(11 \%)$ & 0.07 \\
\hline Median amount of ventricular pacing (IQR) & $30 \%(1-99)$ & $100 \%(99-100)$ & $12 \%(1-82)$ & $<0.01$ \\
\hline Indication for CIED implantation & & & & $<0.01$ \\
\hline Sick sinus syndrome & $196(24 \%)$ & $12(9 \%)$ & $184(27 \%)$ & \\
\hline Second or third-degree AV block & $247(31 \%)$ & $95(73 \%)$ & $152(23 \%)$ & \\
\hline Atrial fibrillation with bradycardia & $125(16 \%)$ & $14(11 \%)$ & $111(17 \%)$ & \\
\hline Carotid sinus syndrome & $2(0.2 \%)$ & $0(0 \%)$ & $2(0.3 \%)$ & \\
\hline Prophylactic $^{c}$ & $232(29 \%)$ & $10(7 \%)$ & $222(33 \%)$ & \\
\hline Implant duration $>5$ years & $330(41 \%)$ & $74(56 \%)$ & $256(38 \%)$ & $<0.01$ \\
\hline \multicolumn{5}{|l|}{ Medication } \\
\hline Beta-blockers & $539(67 \%)$ & $85(65 \%)$ & $454(68 \%)$ & 0.14 \\
\hline Amiodarone & $44(5 \%)$ & $7(5 \%)$ & $37(6 \%)$ & 0.94 \\
\hline Digitalis & $68(8 \%)$ & $9(7 \%)$ & $59(9 \%)$ & 0.58 \\
\hline ACEI & $419(52 \%)$ & $77(59 \%)$ & $342(51 \%)$ & 0.12 \\
\hline Angiotensin receptor blockers & $171(21 \%)$ & $25(19 \%)$ & $146(22 \%)$ & 0.57 \\
\hline Diuretics & $549(68 \%)$ & $100(76 \%)$ & $449(67 \%)$ & 0.04 \\
\hline Aldosterone antagonists & $226(28 \%)$ & $28(21 \%)$ & $198(30 \%)$ & 0.07 \\
\hline Angiotensin-neprilysin inhibitor & $25(3 \%)$ & $1(1 \%)$ & $24(4 \%)$ & 0.16 \\
\hline
\end{tabular}

Plus-minus values are given as mean \pm standard deviation; ACEI — angiotensin converting enzyme inhibitors; AV — atrioventricular; CIED — cardiovascular implantable electronic device; IQR - interquartile range; NYHA - New York Heart Association ${ }^{a} \mathrm{~B}$-type natriuretic peptide was available in only 702 of 802 patients $(88 \%)$

'Other cardiac diseases include hypertrophic cardiomyopathy, cardiac sarcoidosis, cardiac amyloidosis, and tricuspid valve replacement 'Implantable cardioverter-defibrillator without symptomatic bradyarrhythmia at implant 
(70\%) patients with a permanent pacemaker and 239 (30\%) patients with an ICD. Mean duration between device implant and follow-up visit was $67 \pm 70$ months with a minimum implant duration of 6 months. The majority of patients were male (65\%). Mean age at device implant was $74 \pm 13$ years. Indication for pacemaker implantation was a high-degree AV block in 247 (31\%) patients, sick sinus syndrome in 196 (24\%) patients, carotid sinus syndrome in $2(0.2 \%)$ patients, and atrial fibrillation with bradycardia in $125(16 \%)$ patients. CRT devices were implanted in 93 of 802 study patients (12\%) including CRT pacemakers in 25 of 563 pacemaker patients (4\%) and CRT defibrillators in 68 of $239(28 \%)$ patients with an ICD.

\section{Prevalence and predictors of pacing dependency}

A total of 131 of 802 study patients (16\%) were found to be pacing dependent at follow-up $67 \pm 70$ months after the device implant. Pacing dependency at follow-up was found significantly more often in patients with New York Heart Association (NYHA) heart failure class 3 or 4, elevated B-type natriuretic peptide $(\mathrm{BNP})>150 \mathrm{pg} / \mathrm{mL}$, decreased left ventricular ejection fraction (LVEF) $\leq 30 \%$, CKD, high degree AV block at implant, left bundle branch block on electrocardiogram at implant, and implant duration $>5$ years (Table 1 ). Multivariate logistic regression analysis revealed a significant association between pacing dependency and the following 6 clinical variables: second or third-degree AV block at implant (odds ratio $[\mathrm{OR}]=19.9 ; 95 \%$ confidence interval $[\mathrm{CI}]: 10.9-38.5, \mathrm{p}<0.01)$, atrial fibrillation at implant $(\mathrm{OR}=2.15 ; 95 \% \mathrm{CI}$ : $1.16-4.05, \mathrm{p}=0.02), \mathrm{LVEF} \leq 30 \%(\mathrm{OR}=2.06$; 95\% CI: $1.03-4.15, \mathrm{p}=0.04), \mathrm{BNP}>150 \mathrm{pg} / \mathrm{mL}$ $(\mathrm{OR}=2.12 ; 95 \% \mathrm{CI}: 1.16-3.97, \mathrm{p}=0.02), \mathrm{CKD}$ $(\mathrm{OR}=1.86 ; 95 \% \mathrm{CI}=1.08-3.26, \mathrm{p}=0.03)$, and follow-up duration $>5$ years $(\mathrm{OR}=3.29 ; 95 \%$ CI: $1.96-5.64, \mathrm{p}<0.01$ ) (Table 2). None of the remaining clinical variables including age, gender, body mass index, arterial hypertension, diabetes mellitus, underlying heart disease, prior cardiac surgery, transcatheter aortic valve replacement or medication during follow-up including beta-blockers and amiodarone predicted pacing dependency.

\section{Subgroup analysis of 563 patients with permanent pacemaker \\ The results for the subgroup of 563 patients with permanent pacemaker without cardioverter defibrillator back-up are summarized in Table 3 . Pacing dependency at follow-up was found sig-}

nificantly more often in patients with NYHA heart failure class 3 or 4 , elevated BNP $>150 \mathrm{pg} / \mathrm{mL}$, decreased $\mathrm{LVEF} \leq 30 \%$, CKD, second or third-degree $\mathrm{AV}$ block at implant, and implant duration $>5$ years.

Subgroup analysis of 239 patients with ICD

The results for the subgroup of 239 patients with ICD are summarized in Table 4. Pacing dependency at follow-up was found significantly more often in patients with NYHA heart failure class 3 or 4 , elevated BNP $>150 \mathrm{pg} / \mathrm{mL}$, decreased $\mathrm{LVEF} \leq 30 \%$, CKD, non-ischemic dilated cardiomyopathy, amiodarone therapy, and implant duration $>5$ years. In addition, left bundle branch block at implant, which was treated with a CRT defibrillator in 68 patients with ICD, was also associated with a higher prevalence of pacing dependency at followup (Table 4).

\section{Discussion}

The main finding of the present study is a $16 \%$ prevalence of pacing dependency at 67 months mean follow-up, which was associated with second or third-degree AV-block at implant, atrial fibrillation before implant, low LVEF, elevated BNP, CKD and implant duration. Although it is generally accepted that pacing dependency means absence of a sufficient intrinsic rhythm resulting in bradycardia-related symptoms during inhibition of pacing, the definition of pacing dependency is still controversial [12-14]. Similar to previous studies $[5-7,10]$, the current study defined pacing dependency as absence of an intrinsic rhythm of at least $30 \mathrm{bpm}$ during pacemaker inhibition or ventricular pacing at a rate of lower than $30 \mathrm{bpm}$, whereas other investigators used an upper rate cutoff for the intrinsic rhythm of $40 \mathrm{bpm}[4,8,9,11]$ or $50 \mathrm{bpm}$ [3] to define pacing dependency. The observed prevalence of pacing dependency of $16 \%$ in the present study is similar to the prevalence of $22 \%$ in a relatively large Canadian Trial of Physiologic Pacing (CTOPP), in which a pacemaker dependency test was performed in 2244 patients [4]. Of note, the prevalence of pacing dependency varies in the literature between $2 \%$ in the study of Lekalowski et al. [6] and 63\% in the study of Merin et al. [8] as summarized in Table 5 . The discrepancy between these studies may, in part, be explained by differences in study patients as well as different definitions used for pacing dependency. Whereas the current study enrolled consecutive patients who came for routine device follow-up visits at 
Table 2. Results of the multivariate analysis for pacing dependency in 802 study patients.

\begin{tabular}{lcc}
\hline Clinical variable & OR $(95 \% \mathrm{CI})^{\mathrm{a}}$ & P $^{\mathrm{a}}$ \\
\hline Left ventricular ejection fraction $\leq 30 \%$ & $2.06(1.03-4.15)$ & 0.04 \\
B-type natriuretic peptide $>150 \mathrm{pg} / \mathrm{mL}$ & $2.12(1.16-3.97)$ & 0.02 \\
Second- or third-degree AV block at implant & $19.9(10.9-8.5)$ & $<0.01$ \\
Atrial fibrillation before implant & $2.15(1.16-4.05)$ & 0.02 \\
Chronic kidney disease & $1.86(1.08-3.26)$ & 0.03 \\
Implant duration $>5$ years & $3.29(1.96-5.64)$ & $<0.01$ \\
\hline
\end{tabular}

${ }^{a}$ After adjustment for potential confounding clinical variables as summarized in Table 1 including medication, implantable cardioverter defibrillator therapy and cardiac resynchronization therapy; $\mathrm{AV}$ - atrioventricular; $\mathrm{Cl}$ - confidence interval; $\mathrm{OR}$ - odds ratio

Table 3. Subgroup analysis of 563 patients with permanent pacemaker.

\begin{tabular}{|c|c|c|c|c|}
\hline \multirow[t]{2}{*}{ Clinical variable } & \multirow{2}{*}{$\begin{array}{c}\text { All patients } \\
N=563\end{array}$} & \multicolumn{2}{|c|}{ Pacemaker dependency } & \multirow[t]{2}{*}{$\mathbf{P}$} \\
\hline & & Yes $(n=103)$ & No $(n=460)$ & \\
\hline Age [years] & $76 \pm 12$ & $77 \pm 12$ & $76 \pm 12$ & 0.57 \\
\hline Male gender & $336(60 \%)$ & $68(66 \%)$ & $268(58 \%)$ & 0.18 \\
\hline Atrial fibrillation before implant & $194(34 \%)$ & $30(29 \%)$ & $164(36 \%)$ & 0.25 \\
\hline Left bundle branch block at implant & $71(13 \%)$ & $11(11 \%)$ & $60(13 \%)$ & 0.51 \\
\hline Chronic kidney disease & $230(41 \%)$ & $55(53 \%)$ & $175(38 \%)$ & $<0.01$ \\
\hline \multicolumn{5}{|l|}{ Heart failure severity } \\
\hline Left ventricular ejection fraction $\leq 30 \%$ & $60(11 \%)$ & $18(17 \%)$ & $42(9 \%)$ & 0.02 \\
\hline NYHA functional class III or IV & $195(35 \%)$ & $53(51 \%)$ & $142(31 \%)$ & $<0.01$ \\
\hline B-type natriuretic peptide $>150 \mathrm{pg} / \mathrm{mL}^{\mathrm{a}}$ & $264(54 \%)$ & $56(68 \%)$ & $208(51 \%)$ & $<0.01$ \\
\hline Underlying cardiac disease & & & & 0.05 \\
\hline Coronary artery disease & $201(36 \%)$ & $37(37 \%)$ & $164(36 \%)$ & \\
\hline Nonischemic dilated cardiomyopathy & $26(5 \%)$ & $8(8 \%)$ & $18(4 \%)$ & \\
\hline Hypertensive heart disease & $169(30 \%)$ & $35(34 \%)$ & $134(29 \%)$ & \\
\hline Valvular heart disease & $88(16 \%)$ & $16(16 \%)$ & $72(16 \%)$ & \\
\hline Other cardiac diseases ${ }^{b}$ & $5(1 \%)$ & $3(3 \%)$ & $2(0.4 \%)$ & \\
\hline No structural heart disease & $74(13 \%)$ & $4(4 \%)$ & $70(15 \%)$ & \\
\hline \multicolumn{5}{|l|}{ Previous cardiac surgery } \\
\hline Aortocoronary bypass grafting & $55(10 \%)$ & $11(11 \%)$ & $44(10 \%)$ & 0.87 \\
\hline Surgical aortic valve replacement & $20(4 \%)$ & $4(4 \%)$ & $16(3 \%)$ & 0.84 \\
\hline Mitral valve reconstruction or replacement & $12(2 \%)$ & $1(1 \%)$ & $11(2 \%)$ & 0.37 \\
\hline Transcatheter aortic valve replacement & $41(7 \%)$ & $8(8 \%)$ & $33(7 \%)$ & 0.83 \\
\hline Indication for pacemaker implantation & & & & $<0.01$ \\
\hline Sick sinus syndrome & $190(34 \%)$ & $12(12 \%)$ & $178(39 \%)$ & \\
\hline Second or third-degree AV block & $227(40 \%)$ & $79(77 \%)$ & $148(32 \%)$ & \\
\hline Atrial fibrillation with bradycardia & $119(21 \%)$ & $12(12 \%)$ & $107(23 \%)$ & \\
\hline Carotid sinus syndrome & $2(0.4 \%)$ & $0(0 \%)$ & $2(0.4 \%)$ & \\
\hline Cardiac resynchronisation ${ }^{\mathrm{b}}$ & $25(4 \%)$ & $0(0 \%)$ & $25(5 \%)$ & \\
\hline Implant duration $>5$ years & $222(39 \%)$ & $59(57 \%)$ & $163(35 \%)$ & $<0.01$ \\
\hline \multicolumn{5}{|l|}{ Medication } \\
\hline Beta-blockers & $331(59 \%)$ & $59(57 \%)$ & $272(59 \%)$ & 0.47 \\
\hline Amiodarone & $18(3 \%)$ & $0(0 \%)$ & $18(4 \%)$ & 0.08 \\
\hline Digitalis & $46(8 \%)$ & $5(5 \%)$ & $41(9 \%)$ & 0.25 \\
\hline ACEI & $278(43 \%)$ & $58(56 \%)$ & $220(48 \%)$ & 0.15 \\
\hline Angiotensin receptor blockers & $114(20 \%)$ & $18(17 \%)$ & $96(21 \%)$ & 0.52 \\
\hline Diuretics & $362(64 \%)$ & $73(71 \%)$ & $289(63 \%)$ & 0.12 \\
\hline Aldosterone antagonists & $89(16 \%)$ & $12(12 \%)$ & $77(17 \%)$ & 0.26 \\
\hline Angiotensin-neprilysin inhibitor & $9(2 \%)$ & $1(1 \%)$ & $8(2 \%)$ & 0.89 \\
\hline
\end{tabular}

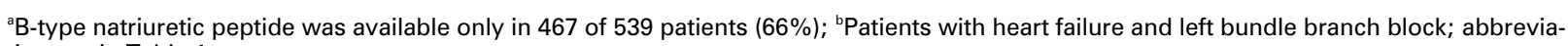
tions as in Table 1. 
Table 4. Subgroup analysis of 239 patients with implantable cardioverter-defibrillator.

\begin{tabular}{|c|c|c|c|c|}
\hline \multirow[t]{2}{*}{ Clinical variable } & \multirow{2}{*}{$\begin{array}{c}\text { All patients } \\
\text { N }=239\end{array}$} & \multicolumn{2}{|c|}{ Pacemaker dependency } & \multirow[t]{2}{*}{$\mathbf{P}$} \\
\hline & & Yes $(n=28)$ & No $(n=211)$ & \\
\hline Age [years] & $68 \pm 12$ & $68 \pm 12$ & $68 \pm 12$ & 0.93 \\
\hline Male gender & $185(77 \%)$ & $21(75 \%)$ & $164(78 \%)$ & 0.81 \\
\hline Atrial fibrillation before implant & $48(20 \%)$ & $8(29 \%)$ & $40(19 \%)$ & 0.41 \\
\hline Left bundle branch block at implant & $70(29 \%)$ & $14(50 \%)$ & $56(25 \%)$ & 0.02 \\
\hline Chronic kidney disease & $100(42 \%)$ & $15(54 \%)$ & $85(40 \%)$ & 0.18 \\
\hline \multicolumn{5}{|l|}{ Heart failure severity } \\
\hline Left ventricular ejection fraction $\leq 30 \%$ & $119(50 \%)$ & $23(82 \%)$ & $96(45 \%)$ & $<0.01$ \\
\hline NYHA functional class III or IV & $125(52 \%)$ & $21(75 \%)$ & $104(49 \%)$ & 0.01 \\
\hline B-type natriuretic peptide $>150 \mathrm{pg} / \mathrm{mL}^{\mathrm{a}}$ & $144(61 \%)$ & $21(78 \%)$ & $123(59 \%)$ & $<0.01$ \\
\hline Underlying cardiac disease & & & & 0.03 \\
\hline Coronary artery disease & $112(47 \%)$ & $8(29 \%)$ & $104(49 \%)$ & \\
\hline Nonischemic dilated cardiomyopathy & $93(39 \%)$ & $18(64 \%)$ & $75(36 \%)$ & \\
\hline Hypertensive heart disease & $11(5 \%)$ & $0(0 \%)$ & $11(5 \%)$ & \\
\hline Valvular heart disease & $13(5 \%)$ & $1(4 \%)$ & $12(6 \%)$ & \\
\hline Other cardiac diseases ${ }^{b}$ & $8(3 \%)$ & $1(4 \%)$ & $7(3 \%)$ & \\
\hline No structural heart disease & $2(1 \%)$ & $0(0 \%)$ & $2(1 \%)$ & \\
\hline \multicolumn{5}{|l|}{ Previous cardiac surgery } \\
\hline Aortocoronary bypass grafting & $40(17 \%)$ & $6(21 \%)$ & $34(16 \%)$ & 0.66 \\
\hline Surgical aortic valve replacement & $9(4 \%)$ & $0(0 \%)$ & $9(4 \%)$ & 0.27 \\
\hline Mitral valve reconstruction or replacement & $3(1 \%)$ & $0(0 \%)$ & $3(1 \%)$ & 1.00 \\
\hline Transcatheter aortic-valve replacement & $2(1 \%)$ & $1(4 \%)$ & $1(0.5 \%)$ & 0.56 \\
\hline \multicolumn{4}{|c|}{ Indication for antibradycardia pacing at ICD implant } & $<0.01$ \\
\hline Sick sinus syndrome & $6(3 \%)$ & $0(0 \%)$ & $6(3 \%)$ & \\
\hline Second or third-degree AV block & $20(8 \%)$ & $16(57 \%)$ & $4(2 \%)$ & \\
\hline Atrial fibrillation with bradycardia & $6(3 \%)$ & $2(7 \%)$ & $4(2 \%)$ & \\
\hline Cardiac resynchronization therapy ${ }^{b}$ & $68(28 \%)$ & $20(71 \%)$ & $48(23 \%)$ & \\
\hline Implant duration $>5$ years & $108(45 \%)$ & $15(54 \%)$ & $93(44 \%)$ & 0.03 \\
\hline \multicolumn{5}{|l|}{ Medication } \\
\hline Beta-blockers & $208(87 \%)$ & $26(93 \%)$ & $182(86 \%)$ & 0.49 \\
\hline Amiodarone & $26(11 \%)$ & $7(25 \%)$ & $19(9 \%)$ & 0.03 \\
\hline Digitalis & $22(9 \%)$ & $4(14 \%)$ & $18(9 \%)$ & 0.52 \\
\hline ACEI & $141(59 \%)$ & $19(68 \%)$ & $122(58 \%)$ & 0.31 \\
\hline Angiotensin receptor blockers & $57(24 \%)$ & $7(25 \%)$ & $50(24 \%)$ & 0.88 \\
\hline Diuretics & $187(78 \%)$ & $27(96 \%)$ & $160(76 \%)$ & 0.03 \\
\hline Aldosterone antagonists & $137(57 \%)$ & $16(57 \%)$ & $121(57 \%)$ & 0.98 \\
\hline Angiotensin-neprilysin inhibitor & $16(7 \%)$ & $0(0 \%)$ & $16(8 \%)$ & 0.27 \\
\hline
\end{tabular}

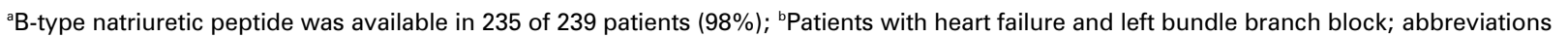
as in Table 1.

the pacemaker and ICD outpatient department, Nagatamo et al. [5] excluded patients with an intrinsic rate of $<30 \mathrm{bpm}$ at implant which likely contributed to a low prevalence of pacing dependency of $4 \%$ in the study by Nagatamo et al. [5]. In contrast to the study by Nagatamo et al. [5] and the present study, Merin et al. [8] found a high prevalence of pacing dependency of $63 \%$ during follow-up. This discrepancy is likely to be due to the fact that the majority of patients in the study of Merin et al. [8] received a permanent pacemaker for third degree AV block without 
Table 5. Prevalence of pacing-dependency in studies with at least 50 patients.

\begin{tabular}{lccccccc}
\hline Author & Year & Patients & ICD & $\begin{array}{c}\text { Cardiac } \\
\text { surgery }\end{array}$ & $\begin{array}{c}\text { Follow-up } \\
\text { [months] }\end{array}$ & $\begin{array}{c}\text { Pacing- } \\
\text { dependency }\end{array}$ & $\begin{array}{c}\text { Intrinsic } \\
\text { rhythm } \\
\text { [bpm] }\end{array}$ \\
\hline Glikson et al. [3] & 1997 & 86 & $0 \%$ & $100 \%$ & 41 (median) & $51(59 \%)$ & $<50$ \\
Tang et al. [4] & 2001 & 2244 & $0 \%$ & NA & 2 to 8 & $484(22 \%)$ & $<40$ \\
Nagatomo et al. [5] & 2004 & 518 & $0 \%$ & NA & $44 \pm 32$ & $23(4 \%)$ & $<30$ \\
Lelakowski et al. [6] & 2007 & 3638 & $0 \%$ & NA & $58 \pm 22$ & $76(2 \%)$ & $<30$ \\
Onalan et al. [7] & 2008 & 102 & $0 \%$ & $100 \%$ & $48($ mean) & $21(23 \%)$ & $<30$ \\
Merin et al. [8] & 2009 & 58 & $0 \%$ & $100 \%$ & $72 \pm 32$ & $37(63 \%)$ & $<40$ \\
Raza et al. [9] & 2011 & 90 & $0 \%$ & $100 \%$ & $67 \pm 50$ & $36(40 \%)$ & $<40$ \\
Rene et al. [10] & 2013 & 98 & $0 \%$ & $100 \%$ & $43 \pm 41$ & $44 \%)$ & $<30$ \\
Sood et al. [11] & 2013 & 1058 & $100 \%$ & NA & $50 \pm 41$ & $142(13 \%)$ & $<40$ \\
Present study & 2019 & 802 & $30 \%$ & $30 \%$ & $67 \pm 70$ & $131(16 \%)$ & $<30$ \\
\hline
\end{tabular}

Intrinsic rhythm used to define pacemaker dependency; 'Intrinsic rhythm $<40 \mathrm{bpm}$ or $<50$ bpm with symptoms; NA - data not available

sufficient ventricular escape rhythm following cardiac surgery [8].

The most important predictor of pacing dependency during follow-up in the current study was the presence second degree AV block type Mobitz or third-degree AV block at the time of pacemaker implant. This is consistent with the findings of most previous studies in patients with and without cardiac surgery prior to pacemaker implant [3, $5-8,15-17]$. Several previous investigators also found an association between pacing dependency during follow-up and body mass index [7], age [11], male gender [11], and a history of coronary artery disease [16]. Whereas none of these variables predicted pacing dependency in the present study, a significant association was found between pacing dependency and heart failure severity as indexed by a low LVEF $\leq 30 \%$ and an elevated BNP level. Furthermore, amiodarone use was associated with pacing dependency in the subgroup of patients with ICD in the current study. This is consistent with the results of Sood et al. [11], who also found a significant association between pacing dependency and amiodarone use in a large cohort of 1058 patients with ICD. The results of the present study support the hypothesis that patients with heart failure and high degree AV block at implant will probably be paced more frequently in the ventricle and may therefore benefit from physiological pacing [4]. Although CKD is a generally accepted important comorbidity in patients with heart disease of any etiology with regard to overall survival, the association between CKD and pacing dependency has not been investigated in previous studies [2-17]. Multivariate analysis in the current study revealed a twofold risk for pacing dependency in patients with CKD compared to patients without CKD.

\section{Limitations of the study}

There are several limitations of the present study. First, clinical patient data and implant data were collected retrospectively, although pacing dependency was determined prospectively between January 2018 and December 2018 at the documented pacemaker and ICD outpatient department. Secondly, pacing dependency was determined during a brief period of time at a single outpatient visit. It is well known that pacing dependency can occur transiently. Therefore, repeated pacing dependency tests and longer monitoring time may have revealed patients, in whom pacing dependency may have resolved or may have occurred at other times.

\section{Conclusions}

In conclusion, pacing dependency after CIED implantation depends on the pacing indication and is much more common in patients with high-degree AV-block at implant compared to patients with sick sinus syndrome. In addition, pacing dependency is associated with more advanced heart failure, CKD and follow-up duration after implant. Since pacing dependent patients who suffer from heart failure will need frequent ventricular pacing, physiological pacing should be considered in these patients.

Conflict of interest: None declared 


\section{References}

1. Brignole M, Auricchio A, Baron-Esquivias G, et al. ESC Committee for Practice Guidelines (CPG), Document Reviewers. 2013 ESC Guidelines on cardiac pacing and cardiac resynchronization therapy: the Task Force on cardiac pacing and resynchronization therapy of the European Society of Cardiology (ESC). Developed in collaboration with the European Heart Rhythm Association (EHRA). Eur Heart J. 2013; 34(29): 2281-2329, doi: 10.1093/ eurheartj/eht150, indexed in Pubmed: 23801822.

2. Steyers CM. 3rd, khera R, bhave P. Pacemaker dependency after cardiac surgery: a systematic review of current evidence. PLoS One. 2015; 10: e0140340.

3. Glikson M, Dearani JA, Hyberger LK, et al. Indications, effectiveness, and long-term dependency in permanent pacing after cardiac surgery. Am J Cardiol. 1997; 80(10): 1309-1313, doi: 10.1016/ s0002-9149(97)00671-1, indexed in Pubmed: 9388104.

4. Tang AS, Roberts RS, Kerr C, et al. Relationship between pacemaker dependency and the effect of pacing mode on cardiovascular outcomes. Circulation. 2001; 103(25): 3081-3085, doi: 10.1161/01.cir.103.25.3081, indexed in Pubmed: 11425772.

5. Nagatomo T, Abe H, Kikuchi K, et al. New onset of pacemaker dependency after permanent pacemaker implantation. Pacing Clin Electrophysiol. 2004; 27(4): 475-479, doi: 10.1111/j.15408159.2004.00466.x, indexed in Pubmed: 15078400.

6. Lelakowski J, Majewski J, Bednarek J, et al. Pacemaker dependency after pacemaker implantation. Cardiol J. 2007; 14(1): 83-86, indexed in Pubmed: 18651439.

7. Onalan O, Crystal A, Lashevsky I, et al. Determinants of pacemaker dependency after coronary and/or mitral or aortic valve surgery with long-term follow-up. Am J Cardiol. 2008; 101(2): 203-208, doi: 10.1016/j.amjcard.2007.07.062, indexed in Pubmed: 18178407.

8. Merin O, Ilan M, Oren A, et al. Permanent pacemaker implantation following cardiac surgery: indications and longterm follow-up. Pacing Clin Electrophysiol. 2009; 32(1):
7-12, doi: 10.1111/j.1540-8159.2009.02170.x, indexed in Pubmed: 19140907.

9. Raza SS, Li JM, John R, et al. Long-term mortality and pacing outcomes of patients with permanent pacemaker implantation after cardiac surgery. Pacing Clin Electrophysiol. 2011; 34(3): 331-338, doi: 10.1111/j.1540-8159.2010.02972.x, indexed in Pubmed: 21208223.

10. Rene AG, Sastry A, Horowitz JM, et al. Recovery of atrioventricular conduction after pacemaker placement following cardiac valvular surgery. J Cardiovasc Electrophysiol. 2013; 24(12): 1383-1387, doi: 10.1111/jce.12260, indexed in Pubmed: 24028584.

11. Sood N, Crespo E, Friedman M, et al. Predictors of pacemaker dependence and pacemaker dependence as a predictor of mortality in patients with implantable cardioverter defibrillator. Pacing Clin Electrophysiol. 2013; 36(8): 945-951, doi: 10.1111/ pace.12164, indexed in Pubmed: 23668483.

12. Korantzopoulos P, Letsas KP, Grekas G, et al. Pacemaker dependency after implantation of electrophysiological devices. Europace. 2009; 11(9): 1151-1155, doi: 10.1093/europace/eup195, indexed in Pubmed: 19617204.

13. Levine PA. Pacemaker dependency after pacemaker implantation. Cardiol J. 2007; 14(3): 318-320, indexed in Pubmed: 18651480.

14. Majewski JP, Lelakowski J. Pacemaker dependency: how should it be defined? Europace. 2018; 20(10): 1708, doi: 10.1093/europace/euy010, indexed in Pubmed: 29518191.

15. Feldman S, Glikson M, Kaplinsky E. Pacemaker dependency after coronary artery bypass. Pacing Clin Electrophysiol. 1992; 15(11 Pt 2): 2037-2040, doi: 10.1111/j.1540-8159.1992. tb03017.x, indexed in Pubmed: 1279595.

16. Mar PL, Angus CR, Kabra R, et al. Perioperative predictors of permanent pacing and long-term dependence following tricuspid valve surgery: a multicentre analysis. Europace. 2017; 19(12): 1988-1993, doi: 10.1093/europace/euw391, indexed in Pubmed: 28073887.

17. Kaplan RM, Yadlapati A, Cantey EP, et al. Conduction recovery following pacemaker implantation after transcatheter aortic valve replacement. Pacing Clin Electrophysiol. 2019; 42(2): 146-152, doi: 10.1111/pace.13579, indexed in Pubmed: 30548869. 